\title{
Mehrsprachigkeit. Defizit oder Potenzial? \\ Nationalideologische Hindernisse im Sprachlernumfeld der däni- schen Minderheit in Südschleswig
}

Daniel Frebel und Rune Steenberg Reyhé (Berlin)

\begin{abstract}
This paper examines language learning in the schools of the Danish minority in northern Germany. The paper shows that the pedagogic approach shaped along a historical narrative of Danish national ideology reflected both in the curriculum and the actual teaching practice, negatively influences the students' language learning. Based on 14 in-depth interviews, participant observation and a deep cultural knowledge of the region and the institutions examined, we develop the following hypothesis.

The ideological constraints connected to the minority's position in the Danish nationalhistorical narrative prevent the students' special learning conditions as non-native Danish speakers outside of Denmark from being taken sufficiently into consideration. The students, in a national spirit, are essentially measured by native standards, which most of them have no chance of living up to. This leads to a constant feeling of deficiency in connection with the Danish language on the part of the students and thus an alienation from it. It further has the feedback-effect of limiting both their language learning and further academic performance.

We conclude that the specific plurilingual background of the learners needs to be taking into consideration, and reflected on by both teachers and students themselves by focusing the teaching practice and curriculum on communication, understanding and self-reflexion. This calls for a more pluralistic, flexible and pragmatic conceptualization of language as a tool shaped by social power relations and an emancipatory reflexion of the students' own position, challenges and choices in this constellation, both of which we argue are of high political relevance way beyond the German-Danish border region.
\end{abstract}

\section{$1 \quad$ Einleitung}

In dieser Arbeit untersuchen wir aus sozial- und gesellschaftswissenschaftlicher Perspektive den Zusammenhang zwischen einer nationalideologisch strukturierten Konzeptualisierung von Sprache sowie der damit in Zusammenhang stehenden Sprachpolitik (cf. Shohamy 2006: 57-62) und dem eigentlichen Sprachlernen von Schülern in einem zwei- oder mehrsprachigen Kontext. ${ }^{1}$

\footnotetext{
${ }^{1}$ Unsere hiesige Verwendung des Begriffs der Mehrsprachigkeit ist sehr breit gedacht. Ohne verneinen zu wollen, dass es signifikante Unterschiede zwischen Zweisprachigkeit und Mehrsprachigkeit geben kann, lehnen wir für diesen Artikel eine strikte Trennung zwischen diesen beiden Termini und den dahinterstehenden Konzepten ab. Wir betrachten Zweisprachigkeit als eine Unterform der Mehrsprachigkeit und da wir ebenfalls LokaldialekLinguistik online 79, 5/2016 - http://dx.doi.org/10.13092/lo.79.3337
}

CC by 3.0 
Wir argumentieren, dass ein offener Blick auf Sprache als sich fortlaufend wandelndes und variables Konstrukt - als diskursive Formation - und eine kritische Entschlüsselung von nationalen Sprachstandards als hegemonial erzeugte Festschreibungen zu einem verbesserten Sprachlernen beiträgt und dabei Hilfestellung leisten könnte, das sprachliche, soziale und kulturelle Potential einer mehrsprachigen Umgebung besser umzusetzen.

Die in diesem Aufsatz formulierten Thesen leiten sich aus einer qualitativen Analyse des Sprachlernkontextes an dänischen Schulen in Schleswig-Holstein von den 1990ern bis heute her. Wir beobachten eine Reihe von Schülern aus deutschen Elternhäusern, die im Laufe ihrer schulischen Karriere die dänische Sprache zwar in ausreichendem Maße lernen um ihren Abschluss zu erlangen, sich aber in dieser Sprache nie wohlfühlen und Dänisch in und außerhalb der Schule zunehmend meiden. Sie fühlen sich beim Dänisch Sprechen durchgehend defizitär und werden dadurch in ihrer Motivation und ihrem Sprachlernen stark behindert. Positive Gegenbeispiele bestätigen diese These, da bei Schülern, die durch Erfolgserlebnisse und erlebte Inklusion eine Art positive Identifizierung mit der dänischen Sprache herstellen, eine weitaus höhere Motivation und bessere Ergebnisse zu beobachten sind. Der negative und demotivierende Effekt einer Entfremdung deutsch-muttersprachlicher Kinder von der dänischen Sprache scheint an der Schule und nicht zuletzt im Klassenzimmer geschaffen zu werden. Laut unseren Interviews und Beobachtungen werden die Kinder früh an einem nationalen Standard gemessen, dem sie nicht gerecht werden können. Dabei wird die Besonderheit und teilweise Begrenztheit ihres Lernumfeldes in Punkto Dänisch nicht ausreichend reflektiert oder in der didaktischen Umsetzung beachtet. Außerdem findet ihre zweisprachige Kompetenz im schulischen Kontext keine Anerkennung und die Schüler erleben dadurch insgesamt eine Abwertung. Die von uns identifizierten zentralen Möglichkeitsbedingungen hierfür sind eine starre, am nationalen Standard orientierte Sprachideologie sowie die nationalideologisch geprägten Institutionen der dänischen Minderheit und die hiermit verbundene besondere Machtdynamik innerhalb des Klassenzimmers.

\section{$2 \quad$ Methodik}

Dieser Aufsatz fasst die Ergebnisse einer ersten Forschung zum Thema zusammen und entwirft die Grundlagen für ein mögliches größeres Forschungsprojekt zum Zusammenhang zwischen Sprachideologie, Sprachkonzeptualisierung und Sprachlernen in der dänischen Minderheit in Südschleswig sowie in mehrsprachigen Kontexten allgemein. Die bisherige Forschung umfasst eingehende Literaturrecherchen, Auswertung von schriftlichen und visuellen Medienquellen samt 14 qualitative unstrukturierte Tiefeninterviews von ein bis drei Stunden Länge. Die Interviews wurden mit früheren oder jetzigen Schülern, Eltern und Lehrern der dänischen Schulen in Südschleswig geführt und hatten eine offene, aber leicht biographische Grundstruktur samt einen thematischen Leitfaden. Mit drei Hauptinformanten wurden jeweils mehrere Interviews mit einer Gesamtlänge von über sechs Stunden geführt. Die Autoren dieses Aufsatzes sind mit dem Lokalmilieu gut vertraut, da beide als Teil der dänischen Minderheit in Südschleswig aufgewachsen sind, in den 1990 und frühen 2000ern die dänischen Schu-

te, Soziolekte und stark den Sprachhabitus prägende soziale Felder (im Sinne Bourdieu's 1982, 1992) als mögliche relevante Sprachkomplexe in diese Aufrechnung einbeziehen, erscheint uns eine klare Trennung zwischen Zwei- und Mehrsprachigkeit weder angemessen noch praktikabel.

ISSN 1615-3014 
len und Institutionen besuchten und einer von ihnen später kurze Zeit an diesen Schulen arbeitete. Da sie jeweils aus deutschen und dänischen Elternhäusern kommen, bieten sie auch in dieser Hinsicht eine ausgeglichene Perspektive.

\section{Symbol-historische Bedeutung der Region Schleswig für das kulturelle Gedächt- nis Dänemarks}

Bei mehrsprachigen Sprechern wird das Verhältnis der Sprachen zueinander von vielen Faktoren beeinflusst. Somit unterscheiden sich die relativen sprachlichen Kompetenzen der Schüler im Deutschen und Dänischen trotz sozialer und kultureller Gemeinsamkeiten stark individuell. Das Verhältnis wird von dem dynamischen Dominanzverhältnis zwischen den Sprachen in verschiedenen sozialen Kontexten und Institutionen geprägt und von der sozialen Position und der individuellen Sprachlernsituation des Lernenden.

Bevor wir unsere Hypothese an individuellen Beispielen verdeutlichen können, ist es daher notwendig, die ideologisch-historischen Voraussetzungen zu skizzieren, um den weiteren Rahmen der Sprachlernsituation nachvollziehen zu können. Hierzu gehört das kollektive Erinnerungsnarrativ der ,Dänischheit' (danskheden cf. Jenkins 2014: 212-221) in Dänemark und seine besondere Verbindungen zur Minderheit in Deutschland.

Da die Geschichte der Region Schleswig sehr komplex ist und es uns hier vorwiegend auf die Erinnerungsfunktion in dem dänischen national-kulturellen Gedächtnis ankommt, konzentrieren wir uns auf einige wenige Daten und historische Ereignisse, die hierfür von zentraler Bedeutung sind. In diesem Zusammenhang unterscheiden wir nicht zwischen einer Erinnerungspraxis der dänischen Minderheit und einer Dänemarks, sondern beleuchten die nationalideologische Bedeutung der Region für das nationale Identitätsnarrativ des modernen dänischen Nationalstaates und wie diese wiederum die Sprachlernsituation im Minderheitenumfeld Südschleswigs beeinflusst.

Die Abgrenzung gegenüber Deutschland gehört spätestens seit 1864 zum ,,politischen und kulturellen Verhalten und Argumentationsmuster“ (Henningsen 2009: 11) Dänemarks. Mit der Niederlage des dänischen Königreichs im Deutsch-Dänischen Krieg fiel nahezu ganz Schleswig an die Siegermächte Deutschland und Österreich. Dieses Jahr kann als Anfangspunkt des Identitätsnarrativs des modernen Dänemarks betrachtet werden. Dänemark verliert seine regionale Machtposition und wandelt sich in diesem Prozess zur konstitutionellen Monarchie. Der dänische Vielvölkerstaat vollzieht eine Wandlung zu einem kulturell und sprachlich zunehmend homogenen Nationalstaat. Es ist dieses Nationalnarrativ des homogenen, demokratischen Nationalstaates, welches bis heute durch die Gedächtnisarbeit von Generationen von Politikern, Lehrern, Journalisten und anderen der Ausgangspunkt des national-kulturellen Gedächtnisses Dänemarks ist. Andere Erinnerungen, die das Narrativ des Homogenen oder der strikten Abgrenzung zum Anderen herausfordern werden weitgehend marginalisiert.

Für die Position der dänischen Minderheit Südschleswigs im nationalen Identitätsnarrativ Dänemarks sind darüber hinaus die Daten 1848/49 und 1920 von Bedeutung. 1848 markiert den Beginn nationalideologisch motivierter militärischer Auseinandersetzungen mit den Deutschen. Im schleswig-holsteinischen Aufstand standen sich Deutschgesinnte und Dänischgesinnte gegenüber. Beide verfolgten das Ziel unter nationalideologischen Prämissen Vorherrschaft über die Region Schleswig zu erlangen. Diese völkerrechtliche Auseinander-

ISSN 1615-3014 
setzung wurde erst 1920 mit einer Volksabstimmung beendet. Schleswig wurde endgültig in ein dänisches Nordschleswig und ein deutsches Südschleswig geteilt (cf. Hansen 2015). 1920 markiert somit den Anfang der heutigen dänischen Minderheit in Südschleswig. Eine historische Verpflichtung Dänemarks gegenüber den im Süden ,Zurückgelassenen“ entwickelte sich ebenfalls aus den Reaktionen auf die Resultate dieser Volksabstimmung (cf. Neergards 1920).

Der Ethnologe Richard Jenkins (2014) identifiziert in seiner Analyse dänischer Nationalidentität des Alltags 5 Themen, welche wesentlich für die ,Dänischheit‘ sind:

1. Homogenität (vor allem auf Sprache und Kultur bezogen)

2. Gleichheit und Bevölkerungsbeteiligung innerhalb dänischer Grenzen

3. Zusammenarbeit mit den skandinavischen Nachbarn

4. Misstrauen und Feindlichkeit gegenüber Deutschland

5. Politische Selbstbestimmung

Die dänische Minderheit in Südschleswig ist trotz ihrer sozial marginalen Bedeutung für Dänemark symbolisch von zentraler Bedeutung für drei dieser Punkte. So ist die Minderheit mit ihrem komplexen bilingualen Hintergrund eine potentielle Bedrohung eines Homogenitätsnarrativs von Sprache und Kultur. Ebenso stört sie potentiell das Abgrenzungsmuster gegenüber Deutschland und die Betonung des Inneren (innerhalb der eigenen Grenzen) gegenüber einem ,bedrohlichen Äußeren'.

Die Institutionen der Minderheit haben dieses Identitätsnarrativ der Dänischheit und ihre eigene Funktion innerhalb dieses Narrativs internalisiert und sehen ihre eigene Minderheitenexistenz mit der Frage verknüpft, inwieweit sie dazu in der Lage sind zur Stabilisierung dieses kulturellen Erinnerns beizutragen. Sie sind ein zentraler Diskursort des dänischen Nationalstaates.

Die Prämissen einer damit verbundenen nationalsymbolischen Sprachrepräsentation üben wesentlichen Einfluss auf die Sprachlernsituation in der dänischen Minderheit in Deutschland aus. Der Unterricht in den Minderheitsschulen unterscheidet sich formal kaum von dem Unterricht einer Schule in Dänemark. Eine spezifische didaktische Ausbildung oder Fortbildung welche Bilingualität und Mehrsprachigkeit mit einbezieht und sich bspw. am Referenzrahmen für Plurale Ansätze für Sprachen und Kultur (REPA) oder dem Gemeinsamen europäischen Referenzrahmen (GeR) orientiert ist erst im Entstehen. Der überwiegende Anteil der Lehrenden in den Institutionen des Dänischen Schulvereins von Südschleswig, der die Kindergärten, Schulen und Tagesinstitutionen betreibt, hat eine herkömmliche pädagogische Ausbildung in Dänemark absolviert. Dies kann subversive Spannungen im Unterricht verursachen, da die Voraussetzungen eines Kindes und Jugendlichen zum Sprachenlernen in der Minderheit nicht vergleichbar sind mit der Situation eines Kindes in Dänemark, dessen Elternteile beide Dänisch als erste Sprache sprechen, und das sich in einem dänischen sozialen Umfeld bewegt. Das Narrativ einer homogenen ,Dänischheit', zu der sie gehören müssen, von der sie aber gleichzeitig quasi per Definition ausgeschlossen werden, kann zu Identitätskonflikten und ungünstigen Machtdynamiken im Klassenraum führen, solange es nicht reflektiert, selbstständig wahrgenommen und kritisch kontextualisiert werden kann. 


\section{$4 \quad$ Was ist eine ,kulturelle Minderheit'?}

Die dänische Minderheit, wie sie heute existiert, ist in ihrer spezifischen Ausprägung überwiegend ein Resultat jüngerer gesellschaftlicher Strömungen und bilateraler Vereinbarungen zwischen Dänemark und Deutschland, jedoch symbolisch vielfältig mit dem Ausgangsnarrativ und dem kulturellen Gedächtnis dänischer Nationalideologie verflochten. Unter diesen Prämissen legitimiert sich die Minderheit daraus, 1920 als Dänen in Deutschland zurückgelassen jedoch nicht aufgegeben worden zu sein (cf. Nergaars 1920).

Die rechtlichen Grundlagen der Minderheitenangelegenheiten zwischen Deutschland und Dänemark wurden 1955 in den Bonn-Kopenhagener Erklärungen geregelt. Hier heißt es: „Das Bekenntnis zur dänischen Nationalität und zur dänischen Kultur ist frei“ und „darf von Amts wegen nicht bestritten oder nachgeprüft werden“ (Deutscher Grenzverein e. V. 1955). Im Minderheitenumfeld spricht man daher von einer kulturellen Minderheit, in der es jedem freigestellt ist über seine Gesinnung und Zugehörigkeit zur Minderheit selbst zu entscheiden. Dänisch als erste Sprache zu sprechen ist somit keine Voraussetzung.

Diese Minderheitendefinition hat jedoch dazu geführt, dass gewissermaßen als Ausgleich das Bekenntnis zur dänischen Nationalität symbolisch ostentativ hervorgehoben wird. Das Bekenntnis wird dabei im Sinne des Narrativs der oben diskutierten ,Dänischheit‘ interpretiert. Da jedoch der überwiegende Teil der Kinder beim Eintreten in die dänischen Institutionen einen deutschsprachigen Hintergrund hat, ergibt sich hieraus eine Situation, in der die Kinder von den eigenen Minderheiteninstitutionen dazu angehalten werden sich mit einer Nationalideologie zu identifizieren, die sie als soziale Akteure mit deutschsprachigem Hintergrund gleichzeitig kategorisch ausschließt.

Um die hierarchische Struktur und asymmetrische Machtkonstellation im Verhältnis zwischen Institution und Kind zu verdeutlichen, ordnen wir den sprachlichen Hintergrund der Kinder vier Typen zu (cf. Becker-Christensen 2014):

A. Kinder bei denen beide Elternteile Standarddänisch (rigsdansk) als erste Sprache sprechen.

B. Kinder bei denen ein Elternteil Standarddänisch (rigsdansk) als erste Sprache spricht und ein Elternteil Deutsch.

C. Kinder bei denen beide Elternteile ausschließlich Deutsch sprechen.

D. Kinder bei denen beide Elternteile überwiegend Deutsch sprechen, jedoch ein Elternteil oder beide das Schulsystem der dänischen Minderheit durchlaufen haben und daher ein regional geprägtes Dänisch (sydslesvigsk) als Zweitsprache sprechen.

Es ist wenig überraschend dass die Typen A und B in der heutigen Minderheit nur einen marginalen Teil ausmachen. Deutsch ist für die Menschen, die in der Region leben die Sprache, die in den meisten Zusammenhängen gesprochen wird. Daher setzten sich die Typen A und B überwiegend aus Kindern zusammen, bei denen ein Elternteil oder beide in Dänemark aufgewachsen und aufgrund einer Anstellung in einer Minderheiteninstitution in die Region gezo- 
gen sind. ${ }^{2}$ Wie wir sehen werden, ergibt sich hieraus im Zusammenhang mit der beschriebenen Nationalideologie ein strukturelles Problem für den Sprachunterricht.

Der überwiegende Teil der Schüler im Schulsystem der dänischen Minderheit besteht also aus Kindern der Kategorie C (cf. Pedersen 2000). Wir beobachten zur Zeit einen prozentualen Anstieg der Kategorie D, da eine zunehmende Anzahl an Absolventen der dänischen Schulen in der Region bleibt und wählt, ihre Kinder ebenfalls an diese Schulen zu geben. Es handelt sich hierbei aber um eine sehr junge und kaum erforschte Entwicklung. Festzuhalten ist, dass der überwiegende Anteil der Kinder Deutsch als erste Sprache spricht, dass der Erstkontakt zum Dänischen überwiegend in den Kindergärten der dänischen Minderheit stattfindet und dass der weitere Kontakt mit der dänischen Sprache primär auf den institutionellen Rahmen der Schule beschränkt bleibt.

Demgegenüber steht ein Unterricht, der die Kinder und Jugendlichen aufgrund von nationaler Sprachideologie generell wie dänische Erstsprecher behandelt und Abweichungen hiervon negativ bewertet. Trotz der Definition einer ,kulturellen Minderheit' gehört dies zum Legitimationsnarrativ der Minderheiteninstitutionen. Diese wird dadurch verstärkt, dass die Führungselite der Institutionen und ein großer Teil der Lehrer selbst keinen Minderheitenhintergrund haben, sondern aus Dänemark kommen und nur aufgrund ihrer Anstellung in den Institutionen in der Region ansässig geworden sind. Somit unterscheidet sich ihre diskursive Position gegenüber dem nationalideologischen Narrativ Dänemarks und ihr Sprachhabitus von dem ihrer Schülerinnen und Schüler. Die Frage, wie sich die Minderheit definiert, und wie sie sich gegenüber diesem nationalideologischen Narrativ Dänemarks positioniert, ist daher eng verknüpft mit den Voraussetzungen der Vermittlungspraxis von Sprache und Sprachbewusstsein.

\section{5 „Eigentlich kann ich gar kein Dänisch“}

Wenden wir uns nun zwei konkreten Fallbeispielen zu, um die Auswirkungen des nationalideologischen Narrativs auf die individuelle Lernsituation der Schüler zu verdeutlichen. Die Beispiele stammen aus den unstrukturierten Tiefeninterviews und bestätigen die Aussagen anderer Interviewpartner sowie die Beobachtungen der Autoren im Minderheitenumfeld:

(1) Eine junge Frau aus Flensburg, deren Eltern beide Deutsch als erste Sprache sprechen, ist an der dänischen Nordseeküste im Urlaub. Sie hat Abitur am dänischen Gymnasium DuborgSkolen gemacht und überlegt in Dänemark zu studieren. Auf einem Campingplatz kauft sie morgens Brötchen - auf Deutsch. Ihre Freundin ist verwundert, „warum sprichst du kein Dänisch“, fragt sie, „du warst doch auf der dänischen Schule.“ Die junge Frau zögert und antwortet dann: „Ach, ich kann ja eigentlich gar kein Dänisch.“

(2) Ein 9-jähriges Mädchen mit einem bildungsbürgerlichen Hintergrund kommt erst in der zweiten Klasse in eine Schule der dänischen Minderheit. Sie hat zuvor nicht den dänischen Kindergarten besucht und spricht Zuhause kein Dänisch, doch verbessert sie ihr Dänisch kontinuierlich. Sie wird von den Lehrern motiviert und für ihre Lernerfolge gelobt. Die Lehrer legen bei ihr nicht die Kriterien eines dänischen Erstsprechers an. Das Mädchen assoziiert die Sprache positiv und schließt bald zu ihren Mitschülern auf.

\footnotetext{
${ }^{2}$ Die Autoren dieses Aufsatzes gehören jeweils zu den Kategorien A (Eltern aus Dänemark in die Region gezogen) und $\mathrm{C}$.
} 
Der Satz „eigentlich kann ich gar kein Dänisch“ aus dem ersten Beispiel spiegelt berichtete Situationen und Gefühle vieler Schüler der dänischen Minderheit in Südschleswig wider. Er zeigt nicht, dass sie objektiv kein Dänisch kann, sondern viel mehr dass sie das Gefühl hat sich nicht auf Dänisch bewähren zu können. Obwohl die junge Frau zwei Jahre lang den dänischen Kindergarten besuchte, 13 Jahre auf dänischen Schulen verbrachte und die Schullaufbahn mit dem Abitur abschloss, ist sie so unsicher im Verwenden der dänischen Sprache, dass sie behauptet diese nicht zu sprechen. Es ist unsere These, dass dies in Teilen auf die Auswirkungen nationalideologischer Einflüsse auf das Lernumfeld zurückzuführen ist. Versuchen wir also die einzelnen Verbindungen nachzuvollziehen.

Ihr Dänisch ist gut genug für ein dänisches Abitur. Sie beherrscht dänische Fachtermini, kann auf Dänisch den Stickstoffkreislauf erklären, mathematische Textaufgaben lösen sowie Aufsätze und Klausuren schreiben. Sie ist aber mit dem Dänischen als Umgangssprache, in der man small talk führt, nicht vertraut und möchte es als Alltagssprache nur ungern erproben, darum kauft sie ihre Brötchen lieber auf Deutsch. Der dänische Verkäufer am Campingplatz, ein junger Mann, der einige Jahre lang Deutsch in der Schule hatte, bevor er es endlich abwählen konnte, und der als Kind auf RTL und SAT1 Zeichentrickfilme guckte, hat zwar einen starken dänischen Akzent, aber keine Scheu vorm Deutschsprechen. Sie, hingegen, fühlt sich nicht wohl damit, in einer alltäglichen Situation Dänisch zu sprechen und vor allem nicht vor einem Dänen, denn durch dänische Muttersprachler, also ihre Lehrer, hat sie immer wieder vorgeführt bekommen, dass sie die Sprache nicht ausreichend gut spricht. Sie fühlt sich bei der Nutzung des Dänischen defizitär und spricht ein distinkt südschleswiges Dänisch mit hörbarem deutschen Akzent, deutscher Idiomatik und einem begrenzten Wortschatz - und sie ist sich dessen schmerzhaft bewusst. Es scheint deutlich, dass sich die negativen Gefühle in Bezug auf die dänische Sprache über einen langen Zeitraum hinweg entwickelt und gefestigt haben, und dass diese auch ihr Sprachlernen beeinträchtigt haben.

Es ist durchaus beeindruckend, was an den dänischen Kindergärten und Schulen geleistet wird - vor allem von den Kindern. Kinder mit überwiegend deutschsprachigem Hintergrund kommen ohne Vorkenntnisse der dänischen Sprache in den dänischen Kindergarten. Trotz der Tatsache, dass sie außerhalb des Unterrichtes kaum Kontakt mit dem Dänischen haben, lernen sie sehr schnell, sich in der Sprache zu bewähren und im Laufe der Schulzeit erlernen viele die Sprache so gut, dass sie die Schullaufbahn mit einem Abitur nach dänischen Kriterien abschließen.

In unseren Interviews zeigt sich aber, dass viele Schüler aus dem Minderheitenumfeld die dänische Sprache nach dem Schulabschluss aufgeben und die Sprache eher negativ assoziieren. Unsere bisherigen Ergebnisse suggerieren, dass viel Potential ungenutzt bleibt, die jeweilige zweite Sprache (Deutsch oder Dänisch, je nach familiärem Hintergrund) umfassend zu erlernen. Die Möglichkeiten, die sich aus dem regionalen, mehrsprachigen Umfeld ergeben, bleiben weitgehend ungenutzt.

Wir haben es nicht mit individuellen, sondern mit strukturbedingten Problemen zu tun. Die Ursachen liegen unseres Erachtens in den Institutionen und in der praktizierten, am nationalen Standard ausgerichteten, monolithischen Sprachideologie, die sie in sich tragen, und die sie den Kindern diskursiv als primäres Bewertungsmaß vermitteln. Es gibt kaum minderheiten- 
spezifische, für die Region erarbeitete Unterrichtsmaterialien und auch im Unterrichtsstil und pädagogischen Ansatz finden die sprachlichen Voraussetzungen der Kinder wenig Beachtung.

In Beispiel 2, dem Beispiel der zugezogenen Schülerin, wird der ideologische Referenzrahmen auf andere Weise deutlich, indem die Lehrenden andere Kriterien ansetzen und somit auf Lernfortschritte statt auf Defizite gegenüber einem Standard fokussieren, der dem Gesamtlernumfeld der Schüler nicht entspricht. Die Erwartungen der Lehrer zu dem neu zugezogenen Mädchen sind anders, außerdem haben sie es hier mit einer nicht Minderheiten-spezifischen Situation zu tun, auf die sie in ihrer Ausbildung besser vorbereitet wurden. Deutlich wird, dass dieses Mädchen aus Lehrerperspektive zunächst einen anderen Hintergrund und Habitus hat, welcher sie vom symbolideologischen Narrativ freistellt. Deutlich wird jedoch auch, dass trotz solcher Erfolge bei den Lehrenden kein Prozess ausgelöst wird, die ideologischen Voraussetzungen generell zu hinterfragen.

\section{Ideologie und Macht im Klassenzimmer}

Versuchen wir nun die Sprachwendung der jungen Frau aus dem ersten Beispiel nachzuvollziehen. Wie auch andere Interviewpartner berichtet sie von einer erlebten sprachlichen Abwertung beim Dänischsprechen. Sie wird von Lehrern an dem Standard eines Muttersprachlers gemessen, dem sie nicht gerecht wird. In ihr verfestigt sich ein Gefühl, dass sie Dänisch nie ausreichend beherrschen wird, und dass ihre Schwierigkeit Standarddänisch wie ihre Lehrer zu sprechen an ihrem eigenen Unvermögen liegt. Sie erlebt die sprachliche Abwertung als eine Abwertung ihrer Person, der sie nur entkommt, indem sie sich vom Dänischen distanziert.

In den Unterrichtspausen wird überwiegend Deutsch gesprochen, Dänisch wird als eine Art reine Lehrer- und Unterrichtssprache etabliert (cf. Pedersen 2000). Aufgrund des Gefühls, unlösbare Sprachdefizite zu haben, berichten viele Schüler von einer Abneigungen zur dänischen Sprache. Eine positive Identifikation findet daher nur mit dem Deutschen statt. Diese führt wiederum zu diskursiven Konflikten mit den Minderheiteninstitutionen, da sie unvereinbar scheint mit dem dänischen nationalideologischen Narrativ.

Wir konnten beobachten, wie Dänisch von einigen Schülern wie eine Satiresprache verwendet wird, die man, außerhalb des Unterrichts, nie versucht ernsthaft zu sprechen, weil schon seit Jahren von Seiten der Autorität ausgeschlossen wurde, dass man sie jeweils angemessen beherrschen wird. Gleichzeitig besteht das Narrativ sowohl bei Lehrern als auch bei Schülern implizit fort, dass man den nationalen Standard eigentlich auf Erstsprachenniveau beherrschen sollte, und dass es am Unvermögen des Schülers oder seiner Eltern liegt, sollte dieses Ziel verfehlt werden. Die angebotenen Hilfsstellungen in den Institutionen werden daher individuell und nicht strukturell angesetzt und sind wiederum mit einer impliziten Abwertung verbunden.

Auf der anderen Seite werden viele Schüler mit Dänisch als erster Sprache nicht dazu motiviert, Deutsch wirklich gründlich zu erlernen. In den Institutionen wird dem Deutschen und auch der Zweisprachigkeit kaum Wert zugeschrieben. Hochdeutsch wie eine Erstsprache zu beherrschen, könnte sogar ihre Rolle als Vertreter Dänemarks und der dänischen Sprache gefährden. Die in Dänemark aufgewachsenen Eltern und Lehrer sind wenig motiviert, ihre Kinder zum intensiven Deutschlernen anzuhalten oder sich stark mit der Region zu identifizieren, 
da sie davon ausgehen irgendwann nach Dänemark zurückzukehren und auf jeden Fall, dass ihre Kinder es tun. Trotz ihrer Arbeitsstelle in Südschleswig wohnen viele von ihnen weiterhin auf der dänischen Seite der Grenze.

Die junge Frau aus dem Beispiel 1 hat verhältnismäßig wenig Sprachpraxis erlangt, da sie nur im Unterricht die Möglichkeit hatte Dänisch zu sprechen. Hier hielt sie es jedoch, nach eigenen Aussagen, für besser möglichst wenig zu sprechen, um so ihre Sprachdefizite zu verbergen. Das hat sicherlich nicht nur ihre sprachliche, sondern auch ihre allgemeine schulische Entwicklung beeinträchtigt.

An diesen zwei stark vereinfacht wiedergegebenen sozialen Referenzbeispielen wird deutlich, welchen konkreten Einfluss das nationalideologische Narrativ und die damit verbundene monolithische Konzeptualisierung von Sprache auf ein mehrsprachiges Sprachlernumfeld haben kann. Der linguistisch widerlegte Mythos von Sprache als geschlossenem Container (cf. Chomsky 1988) und die damit verbundene Idee perfekter Sprachbeherrschung wird dennoch in vielen Institutionen aufrecht erhalten. So auch der Glaube, dass die besten Ergebnisse durch einseitigen Fokus auf die Zielsprache, definiert entlang des nationalen Standards (hier rigsdansk), erreicht werden können, obwohl die jahrzehntelange praktische Unterrichtserfahrung in Südschleswig die Möglichkeit böte, die Institutionen zu Fürsprechern und Expertenorganisationen von mehrsprachiger Didaktik zu entwickeln.

Man wird heute in Dänemark oder Deutschland kaum Sprachwissenschaftler, Lehrer oder Pädagogen treffen, die individuelle Mehrsprachigkeit als schädlich für die Persönlichkeitsentwicklung und den Bildungsverlauf bezeichnen würden. Im Gegenteil erleben wir im Moment eher einen Trend, der die Vorteile von Plurilingualität zu übersteigern scheint. Auch in Presseberichten über die dänische Minderheit in Südschleswig und in ihrer Selbstdarstellung wird mit ,multikultureller Kompetenz' und ,bilingualen Fähigkeiten“ geworben. Betrachtet man die pädagogische Ausbildung, Fachliteratur, das Curriculum und die verankerte Sprachlernpolitik fällt jedoch auf, dass Mehrsprachigkeit mit ihren Vorteilen und Herausforderungen kaum Beachtung und noch weniger Anerkennung findet.

\section{Vorschläge für das Curriculum}

Die dänische Sprache ist in Südschleswig heute nicht bedroht. Die über Jahrzehnte durch den dänischen Staat getätigten Investitionen und die Bereitschaft der Eltern, ihre Kinder in die Hände dänischer Bildungseinrichtungen zu geben, haben dazu geführt, dass heute weit mehr Menschen in der Region Dänisch beherrschen als vor 40 oder 60 Jahren (cf. BeckerChristensen 2014), wenn auch überwiegend als Zweitsprache. Die politische Emanzipation der Minderheit und das beachtliche akkumulierte sprachliche und kulturelle Kapital (cf. Bourdieu 1992: 45-60) sollte genutzt werden, um das Lernumfeld umzustrukturieren. Ein nationaler Sprachstandard ist nicht die einzige Möglichkeit, Sprache qualitativ zu bewerten. Vielfalt, Kreativität, das spielerische Erproben metaphorischer und grammatischer Grenzen können befördert und belohnt werden statt sanktioniert. Ein starrer und rigider Fokus auf das Einhalten von Standards in der Form von Sprachkorrektheit kann für das Sprachlernen demotivierend und somit äußerst kontraproduktiv sein.

Es besteht kein Zweifel daran, dass man eine Sprache in Bezug auf Ausdrucks- und Lesefähigkeit besser oder schlechter beherrschen kann, und dass nicht jede Missachtung des Stan- 
dards als kreative Erweiterung zu verstehen ist. Auch geht es uns bei diesem Ansatz gewiss nicht darum, die Orientierung am nationalen Standard im Curriculum gänzlich aufzugeben dies wäre auch kaum umsetzbar. Allerdings sollte die Konstruiert- und Begrenztheit eines solch standardisierten Sprachverständisses reflektiert und erörtert und die Dominanz der Standardsprache somit kontextuell verstanden und relativiert werden. So kann ein produktiveres Sprachlernumfeld geschaffen werden, in dem ein Beherrschen des nationalen Standards oder eines besonderen Soziolekts als zugangsgebendes Werkzeug verstanden werden kann, das jeder sich erarbeiten kann, zu dem aber einige durch ihr Umfeld bedingt einen leichteren Weg haben. Somit werden individuelle Schwächen und Stärken strukturell nachvollziehbar, statt an persönliches Unvermögen geknüpft zu werden und sowohl Sprachhabitus (cf. Bourdieu 1982: 171-210) als auch Subjektivierung (cf. Yildiz 2012: 48-55) des Einzelnen insgesamt positiv beeinflusst.

Unsere Forschung suggeriert, dass es dafür empfehlenswert ist, gerade in einem mehrsprachigen Kontext, Sprachkorrektheit vor allem in den unteren Klassen eine untergeordnete Rolle spielen zu lassen. Falls die Förderung interkultureller und bilingualer Fähigkeiten der dänischen Minderheit ein ernstes Anliegen ist, sollte dies im Curriculum verankert werden. Dabei gehören für den Lernprozess vor allem Förderung von Sprachbewusstsein und die Interrelation von Sprache und Kultur in den Vordergrund gestellt. Mit dieser Erkenntnis lassen sich für ein produktives Sprachlernumfeld zwei Kernbereiche identifizieren (cf. Möllering 2014: 65):

Kommunikation: Anstatt zu sehr auf Sprachkorrektheit zu bestehen, sollte der kommunikative und pragmatische Aspekt von Sprache für die Interpretation, Produktion und den Austausch von Bedeutung im Vordergrund stehen. Sprache wird gesehen als ein Zugang zur Welt und zu Möglichkeiten und kann dementsprechend bewusst geübt und geschliffen werden.

Reflektieren: Die Schüler sollten Hilfestellungen erhalten um sich ihrer eigenen Position als Sprechende und Sprachlernende im Verhältnis zur Sprache, anderen Sprechern und den umgebenden Institutionen, Narrativen und Ideologien bewusst zu werden. So lernen sie ihre eigene Sprachbeherrschung als das Ergebnis ihres Umfeldes und ihrer bewussten oder unbewussten Wahlen verstehen - sowohl um aus der Vergangenheit heraus ihre heutige Position zu verstehen als auch um für die Zukunft bewusste Wahlen treffen zu können. Dies setzt voraus, so urteilsfrei wie möglich verstehen zu lernen, wie das eigene Sprachvermögen entstanden ist und wie es bewusst verbessert werden kann.

Insbesondere das zweite Themenfeld sehen wir vernachlässigt. Hier sind Materialien zu folgenden Punkten im Unterricht zu entwickeln und zu erproben (cf. Möllering 2014: 67):

- Linguistische und kulturelle Praktiken als solche erkennen, interpretieren und beschreiben - auch die exkludierenden.

- Vergleich linguistischer und kultureller Systeme einer Sprache zu denen einer anderen und dabei bestehende Ideen und Praktiken in Relation zu anderen hinterfragen.

- Die eigene sprachliche Perspektive wertfrei mit der Anderer abgleichen und das daraus resultierende Wissen produktiv anwenden.

- Ein Bewusstsein dafür entwickeln, wie Sprache und kulturelle Narrativen Identität, Habitus und Subjektivierung beeinflussen. 
- Übersetzung als Handwerk und als Weg zur sprachlichen Selbstreflexion üben.

Sprachbewusstsein, transkulturelle Kompetenzen und tiefe bilinguale Fähigkeiten sind nicht das Gleiche, wie zwei Sprachen mehr oder weniger gut zu sprechen und sie müssen dementsprechend gefördert werden. Der gemeinsame europäische Referenzrahmen für Sprachen (GeR) und der Referenzrahmen für plurale Ansätze zu Sprachen und Kulturen (REPA) liefern bereits gute Ansätze dafür, kulturelle und soziale Phänomene in didaktische Strategien miteinzubeziehen (cf. Meißner 2013; Candelier et al. 2012). Es mangelt jedoch vielerorts daran, diese Ansätze umfassend in der Praxis zu erproben.

\section{Schlussfolgerungen}

Im Fall der dänischen Schulen in Südschleswig wird der vielschichtige Einfluss deutlich, den Ideologien, nationalkulturelles Erinnern (cf. Assman 2013) und symbolische Identitätsnarrativen auf die tägliche Sprachlernsituation in einem Minderheitenumfeld haben können. Wir sind der Überzeugung, dass trotz des spezifischen und privilegierten Kontextes, in dem sich die dänische Minderheit in Deutschland befindet, sich unsere Erkenntnisse und generellen Schlüsse auch auf andere mehrsprachige Kontexte überführen lassen. Das gilt nicht zuletzt für die von uns beobachteten negativen Auswirkungen einer nationalideologisch strukturierten monolithischen Konzeptualisierung von Sprache auf den Erfolg des Sprachlernens und die sprachliche Integration von Kindern, deren erste Sprache nicht die offizielle nationale oder institutionelle Sprache ist. Wir vermuten, dass der geringe Sprachlernerfolg vieler sozial marginalisierter Gruppen in Europa zu einem nicht geringen Teil auf eine solche institutionelle Sprachpraxis zurückzuführen ist, die in einem monolinguistischen Fokus auf Sprachkorrektheit verharrt. Die unterschiedlichen sprachlichen Hintergründe und Lernvoraussetzungen der Kinder und Jugendlichen werden so zu wenig reflektiert und vorwiegend als Problem betrachtet. Wir möchten Anstöße dafür liefern, dies mit recht simplen Mitteln umzukehren und den Fokus auf das Potential von Mehrsprachigkeit zu legen. Unser Anhaltspunkt ist es dabei, die gängigen Konzeptualisierungen von Sprache zu hinterfragen und die Kinder und Jugendlichen zur kritischen Reflexion ihres eigenen sprachlichen Habitus und der damit verbundenen sozialen Position anzuregen. Dies ist effektiv ein emanzipatorischer Ansatz. Ein offeneres Verständnis von Sprache als dynamische Formation, die von Machtstrukturen geprägt ist, ermöglicht den Schülerinnen und Schülern ein tiefes und unsanktioniertes Verstehen ihrer eigenen Sprachlernsituation. Die erlangte Sprachfertigkeit sowie die kontextabhängigen Herausforderungen können in diesem Rahmen befreit von hegemonieller Bewertung besser reflektiert, besprochen und angegangen werden. Den Schülerinnen und Schülern wird ein alternativer Bewertungsraum ermöglicht, der es ihnen erlaubt, ein umfassendes Sprachbewusstsein (cf. Eichler/Nold 2007: 63-82) zu entwickeln und ihnen zu mehr Selbstbestimmung in der individuellen Sprachlernsituation verhilft. Dies ist, unseres Erachtens, der beste Weg zu einem bewussten und ergiebigen Arbeiten mit den eigenen Sprachfähigkeiten.

Eine solche Selbstreflexion und sprachliche Emanzipation zu ermöglichen ist in erster Linie eine Aufgabe, welche sich an diejenigen Institutionen richtet, die intensiven Kontakt mit Kindern und Jugendlichen aus einem mehrsprachigen Umfeld haben. Dazu zählen v. a. Schulen, aber auch Kindergärten, Horte, Jugendzentren, Sportvereine und andere Jugendeinrichtungen. Viele dieser Institutionen sind sich dieser Verantwortung nicht ausreichend bewusst. Durch

ISSN $1615-3014$ 
unreflektierte Konzeptualisierung von Sprache entlang nationaler Standards können Bewertungshierachien aufgebaut werden, die von der Sprache auf den Sprecher projiziert und von diesem internalisiert werden und somit Sprachbarrieren aufbauen oder erhalten, die die Entwicklung eines reflektierten Sprachbewusstseins und letztendlich motiviertes Sprachlernen verhindern. Hiervon sind vor allem Minderheiten, Menschen mit Migrationshintergrund, Asylanten und Bewohner von Grenzgebieten oder Gebieten mit starken Lokalsprachen oder starker Ausprägung eines bildungsfernen oder anders sozial abgewerteten Sprachhabitus betroffen. Es handelt sich also - in Deutschland wie in Dänemark und außerhalb - um eine beachtliche und wachsende Anzahl von Menschen.

\section{$9 \quad$ Ausblick}

Wir haben in diesem Aufsatz einige Aspekte der komplexen Bedingungen beleuchtet, welche ein produktiveres Sprachlernumfeld in der dänischen Minderheit in Südschleswig behindern. Da es sich um eine sehr komplexe Problematik handelt, zu dessen Lösung interdisziplinäres Arbeiten und umfangreiche Datenerhebung notwendig sind, konnten wir unsere Thesen hier nur kurz umreißen.

Eine so vereinfachte Darstellung läßt viele Aspekte unberücksichtigt. So auch die bemerkenswerte Dynamik, die in den letzten Jahren sowohl im Minderheitenumfeld als auch in Dänemark zu beobachten ist. Bestehende nationalideologische Narrative werden hinterfragt und diskursiv verhandelt. Jüngere Generationen der dänischen Minderheit weigern sich zunehmend, nationalideologisch instrumentalisiert zu werden und wählen selbstbestimmt eine lokale Identifikation als Südschleswiger, was auch einen selbstbestimmteren Umgang mit den eigenen Sprachen umfasst.

Doch diese Prozesse sind keineswegs vollbracht und viele institutionelle Barrieren hindern nach wie vor ein volleres Ausschöpfen des Potenzials von Mehrsprachigkeit in Südschleswig und anderen Gebieten und Gruppen in Deutschland, Dänemark und Europa. Wir hoffen in Zukunft durch weitere, umfassendere Forschung die Komplexität der Problematik besser einfangen und zu Lösungen beitragen zu können.

\section{Literatur}

Assmann, Jan (2013): Erinnerung und politische Identität in früheren Hochkulturen. München: Beck.

Becker-Christensen, Henrik (2014): Sproglige forholdi det danske mindretal. www.graenseforeningen.dk/sproglige-forhold-i-det-danske-mindretal-i-sydslesvig.html [02.04.2015].

Bourdieu, Pierre (1982): Die feinen Unterschiede. Kritik der gesellschaftlichen Urteilskraft. Frankfurt a. M.: Suhrkamp.

Bourdieu, Pierre (1992): Die verborgenen Mechanismen der Macht. Schriften zu Politik und Kultur. Herausgegeben von Steinrücke/Margareta, übersetzt aus dem Französischem Bolder, Jürgen/ Nordmann, Ulrike. Hamburg: VSA.

Candelier, Michel et al. (2012): FREPA. A Framework of Reference for Pluralistic Approaches to Languages and Cultures. Competences and Resources. Graz: ECML.

Chomsky, Noam (1988): Language and Problems of Knowledge: The Managua Lectures. Cambridge/MA.: MIT.

ISSN 1615-3014 
Deutscher Grenzverein e. V. (ed.) (1985): Die Bonn-Kopenhagener Erklärungen von 1955. Zur Entstehung eines Modells für nationale Minderheiten. Flensburg: Deutscher Grenzverein e. V.

Eichler, Wolfgang/Nold, Günter (2007): „Sprachbewusstheit““. In: Beck, Bärbel/Klieme, Eckhard (eds.): Sprachliche Kompetenzen. Konzepte und Messung. DESI-Studie. Weinheim/Basel, Beltz: 63-82.

Europarat (2001): Gemeinsamer europäischer Referenzrahmen für Sprachen: lernen, lehren, beurteilen. Berlin: Langenscheidt.

Hansen, Hans Schulz (2014): Sønderjyllands historie. www.graenseforeningen.dk/?q=kortversion.html [02.04.2015].

Henningsen, Bernd (2009): Dänemark. München: Beck.

Jenkins, Richard (2014): At voere dansk - Identitet i hverdagslivet. Kopenhagen: Museum Tuscuanums.

Meißner, Franz-Joseph (2013): Die REPA-Deskriptoren der, weichen' Kompetenzen. Eine praktische Handreichung für den kompetenzorientierten Unterricht zur Förderung von Sprachlernkompetenz, interkulturellem Lernen und Mehrsprachigkeit. Giessen: Giessener Elektronische Bibliothek.

Möllering, Martina (2014): “Australian Language Policy”. In: Grommes, Patrick/Hu, Adelheid (eds.): Plurilingual Education: Policies - Practice - Language Development. Amsterdam, John Benjamins: 55-73.

Neergaards, Nils (1920): „Tale på Dybbøl 11. juli 1920“. In: Graenseforeningens Leksikon www.graenseforeningen.dk/leksikon/s/all/5773 [02.04.2015].

Pedersen, Karen Magrethe (2000): Dansk sprog i Sydslesvig. Det danske sprogs status inden for det danske mindretal $i$ Sydslesvig (bind 1 \& 2). Aabenraa: Institut for Grænseregionsforskning.

Shohamy, Elena (2006): Language Policy: Hidden Agendas and New Approaches. London: Routledge.

Yildiz, Safiye (2012): Interkulturelle Erziehung und Pädagogik: Subjektivierung und Macht in den Ordnungen des nationalen Diskurses. Wiesbaden: Springer. 